\title{
Cerebellar Posterior Superior Vermis and Cognitive Cluster Scores in Drug-Naive Patients with First-Episode Schizophrenia
}

\author{
Gaku Okugawa Kenji Nobuhara Katsunori Takase Toshihiko Kinoshita
}

Department of Neuropsychiatry, Kansai Medical University, Osaka, Japan

\section{Key Words}

Schizophrenia $\cdot$ Vermis, cerebellar $\cdot$ Cognitive cluster scores

\begin{abstract}
Previously, we performed an MRI study that revealed smaller volumes of the subregions of the cerebellar vermis in men and women with chronic schizophrenia. An issue that arose from that study was whether similar structural changes in the cerebellum are found in patients with first-episode schizophrenia. In the present study, MRI scans were acquired from 14 drug-naive patients with first-episode schizophrenia and 16 healthy subjects, and used to measure the volumes of their cerebellar subregions. Positive symptom, negative symptom and cognitive cluster scores were attained using the Positive and Negative Syndrome Scale. Patients with first-episode schizophrenia had reduced volumes of the anterior vermis and posterior superior vermis compared with healthy subjects. We confirmed that there was a volume reduction of the cerebellar vermis in drug-naive patients with first-episode schizophrenia. Smaller volumes of the posterior superior vermis were associated with worse cognitive cluster scores in patients with first-episode schizophrenia.

Copyright ๑ 2008 S. Karger AG, Basel
\end{abstract}

\section{Introduction}

Previous MRI studies have revealed that schizophrenic patients have reduced volumes of the vermal subregions [1-7]. Furthermore, a reduced size of the posterior vermis was found to be associated with cognitive dysfunction in fragile X syndrome [8]. We detected a volume reduction in the cerebellar vermis of men with chronic schizophrenia in a previous study [9]. To investigate the hypothesis that similar structural abnormalities are present in the cerebellar vermis of drug-naive patients with first-episode schizophrenia, we performed MRI scans to compare the volumes of the cerebellar vermis between schizophrenic patients and healthy subjects. We also investigated whether the volumes of the vermal subregions were correlated with the Positive and Negative Syndrome Scale (PANSS) cognitive cluster scores in the patients.

\section{Methods}

\section{Participants}

All procedures were approved by the Institutional Review Board of Kansai Medical University. Written informed consent was obtained from all the participants. A total of 14 patients with schizophrenia (7 males and 7 females) fulfilling the DSM-IV criteria [10] and 16 healthy subjects ( 8 males and 8 females) were

\section{KARGER}

Fax +4161306 1234 E-Mail karger@karger.ch www.karger.com
Gaku Okugawa, MD, PhD

Department of Neuropsychiatry, Kansai Medical University

2-3-1 Shinmachi Hirakata

Osaka 573-1191 (Japan)

Tel. +81 72804 0101, Fax +81 72804 0166, E-Mail okugawa@hirakata.kmu.ac.jp 
Table 1. Relative volumes of cerebellar regions

\begin{tabular}{|c|c|c|}
\hline Cerebellar subregions & $\begin{array}{l}\text { Schizophrenic } \\
\text { subjects } \\
\text { ( } 7 \text { male, } 7 \text { female) }\end{array}$ & $\begin{array}{l}\text { Healthy } \\
\text { subjects } \\
\text { (8 male, } 8 \text { female) }\end{array}$ \\
\hline Right cerebellar hemisphere & $3.95 \pm 0.41$ & $3.91 \pm 0.22$ \\
\hline Left cerebellar hemisphere & $3.94 \pm 0.49$ & $3.92 \pm 0.25$ \\
\hline Cerebellar vermis $^{\mathrm{a}}$ & $0.55 \pm 0.09$ & $0.67 \pm 0.09$ \\
\hline Anterior vermis ${ }^{\mathrm{b}}$ & $0.24 \pm 0.05$ & $0.30 \pm 0.05$ \\
\hline Posterior superior vermis ${ }^{a}$ & $0.16 \pm 0.03$ & $0.21 \pm 0.04$ \\
\hline Posterior inferior vermis & $0.15 \pm 0.03$ & $0.17 \pm 0.03$ \\
\hline
\end{tabular}

Values are means \pm SD.

${ }^{\mathrm{a}} \mathrm{p}=0.001,{ }^{\mathrm{b}} \mathrm{p}=0.005$, Student's t test. missure/posterior commissure line and the interhemispheric fissure was aligned on the other two axes. The $\mathrm{T}_{2}$-weighted images were aligned to the spatially normalized $\mathrm{T}_{1}$-weighted image. The data sets were then segmented into grey matter, white matter and cerebral spinal fluid using a multispectral data analysis method based on automated training class selection [15]. The reproducibility and reliability of the segmentation procedure were previously confirmed $[16,17]$. The intracranial volume was measured by the BRAINS program. The anterior vermis, posterior superior vermis, posterior inferior vermis and cerebellar hemispheres were traced manually and their volumes were measured. The inter- and intraoperator reliabilities for measuring the volumes of the cerebellar subregions were established in a previous study [9]. The current intraclass correlation coefficients [18] of the measured volumes of the cerebellar subregions were above 0.95 .

\section{Statistical Analysis}

Student's $t$ test was used for the assessment of group differences in the relative volumes $(100 \times$ absolute volume/intracranial volume). Pearson's correlation method was used to test the correlations between the relative volumes of regions of interest and the PANSS positive symptom, negative symptom and cognitive cluster scores (difficulty in abstract thinking, stereotyped thinking, cognitive disorganization, lack of judgment and insight, poor attention, tension, mannerisms and posturing) in the schizophrenic patients. Differences were considered to be statistically significant at $\mathrm{p}<0.01$. serious neurological or endocrine disorders, alcohol-related disorders, any medical condition or treatment known to affect the brain, or mental retardation defined according to the DSM-IV criteria. The severity of positive and negative symptoms were assessed using the PANSS, in which higher scores are indicative of worse performance [11]. We measured the PANSS cognitive cluster scores (difficulty in abstract thinking, stereotyped thinking, cognitive disorganization, lack of judgment and insight, poor attention, tension, mannerisms and posturing) in the schizophrenic patients [12].

\section{MRI Scans}

The participants were examined using a 1.5 Tesla GE Signa system (GE Medical, Milwaukee, Wisc., USA) at the Department of Radiology, Kansai Medical University Hospital. $\mathrm{T}_{1}$-weighted images, using a spoiled GRASS sequence, were acquired with the following parameters: $1.5-\mathrm{mm}$ coronal slices; $35^{\circ}$ flip angle; 24 $\mathrm{ms}$ repetition time; $6.0-\mathrm{ms}$ echo time; number of excitations $=1$; 24 -cm field of view; $256 \times 192$ acquisition matrix. $\mathrm{T}_{2}$-weighted images were acquired with the following parameters: $3.0-\mathrm{mm}$ coronal slices; 84-ms echo time; 6,000-ms repetition time; number of excitations $=1 ; 24-\mathrm{cm}$ field of view; $256 \times 192$ acquisition matrix. From visual inspection, all scans were judged to be excellent without any obvious motion artifacts. All scans were evaluated for clinical pathology by a neuroradiologist. No scans were viewed as abnormal from a clinical standpoint. The quantitative analysis was performed in a blinded manner with regard to the 2 diagnostic categories.

\section{Volume Measurements}

MRI data analysis was performed using the BRAINS software $[13,14]$. The $T_{1}$-weighted images were spatially normalized and resampled to $1.0-\mathrm{mm}^{3}$ voxels, so that the anterior-posterior axis of the brain was realigned to become parallel to the anterior com-

\section{Results}

The mean ages of the participants $( \pm$ SD) were $29.8 \pm$ 6.3 and $29.8 \pm 4.3$ years for the schizophrenic patients and healthy subjects, respectively. The mean age at the onset of schizophrenia was $28.1 \pm 5.8$ years among the patients. The mean positive symptom, negative symptom and cognitive cluster scores were $20.0 \pm 6.0,21.9 \pm 5.5$ and $30.1 \pm 3.6$, respectively.

There were significant differences in the relative volumes of the anterior vermis, posterior superior vermis and total vermis between drug-naive patients with firstepisode schizophrenia and healthy subjects (table 1). There were no significant differences in the relative volumes of the posterior inferior vermis and right and left cerebellar hemispheres between schizophrenic patients and healthy subjects.

The relative volumes of the posterior superior vermis were significantly correlated with the PANSS cognitive cluster scores in patients with schizophrenia (fig. 1). There were no significant correlations between any other relative volumes of regions of interest (anterior vermis, posterior inferior vermis and cerebellar hemispheres) and the PANSS positive symptom, negative symptom and cognitive cluster scores in the patients. 


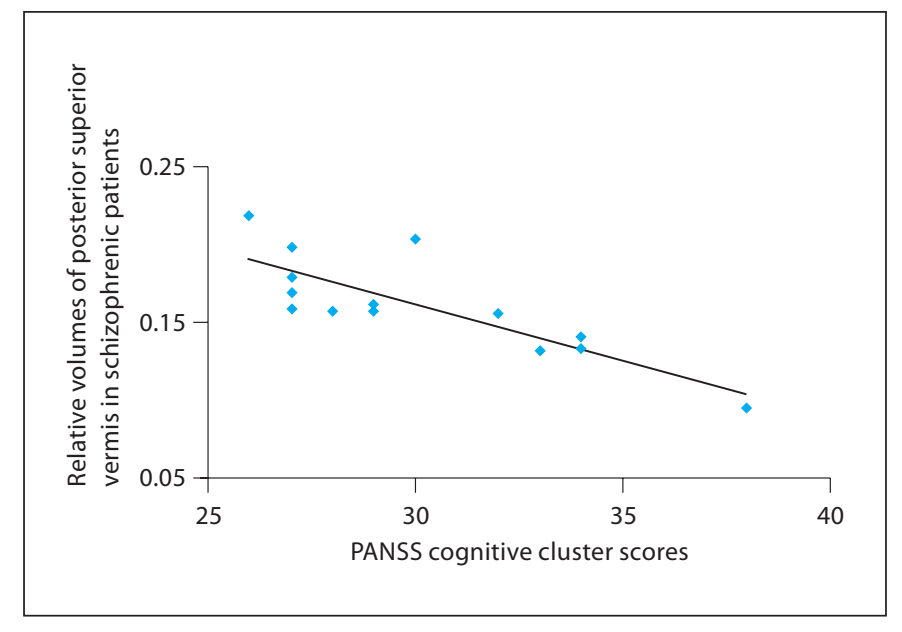

Fig. 1. Correlation between the volumes of the posterior superior vermis and the PANNS cognitive cluster scores in 14 patients with schizophrenia $(\mathrm{r}=-0.82, \mathrm{p}<0.001)$.

\section{Discussion}

Drug-naive patients with first-episode schizophrenia had smaller volumes of the anterior vermis and posterior superior vermis than healthy subjects. Smaller volumes of the posterior superior vermis were associated with worse cognitive cluster scores in drug-naive patients with first-episode schizophrenia.

Alcoholism has been shown to influence cerebellar volume as evidenced by a volume reduction of the anterior vermis in men with alcoholism [19]. Men with alcohol dependence were found to have reduced volumes of the anterior vermis and posterior superior vermis [20]. Alcohol is known to affect cerebellar vermal volumes and cognitive function. Schizophrenic patients who drank no or little alcohol were enrolled in the present study.

In a previous study, patients with chronic schizophrenia had reduced volumes of the anterior vermis, posterior superior vermis and posterior inferior vermis [21]. In the present study, schizophrenic patients had reduced volumes of the anterior vermis and posterior superior vermis at the first episode. The cerebellar volume has been reported to become smaller over time in schizophrenic patients [22]. Since the volumes of the cerebellar structures become smaller during the illness, chronic schizophrenic patients may have reduced volumes of the anterior vermis, posterior superior vermis and posterior inferior vermis. We speculate that the volumes of the cerebellar vermal subregions are reduced at the age of onset in patients with schizophrenia and that these volumes may become smaller over time. Since the present study was a cross-sectional analysis, further longitudinal studies will be necessary to determine the progressive changes in the cerebellar vermal volumes.

With regard to cognitive function, reduced volume of the posterior superior vermis was significantly associated with cognitive cluster scores in schizophrenic patients. Ichimiya et al. [23] described how a reduction in the vermal volume was correlated with Brief Psychiatric Rating Scale 'depression' and 'paranoid' subscores. Therefore, volume reduction of the cerebellar vermis may be one of pathophysiologies in schizophrenia. There were no significant correlations between the volumes of the cerebellar hemispheres and the cognitive cluster scores. Since the mean age at onset for schizophrenia in the present study was quite high at 28 years of age, this may represent a partial reason for the lack of positive correlations between the cerebellar hemispheric volumes and the PANSS subscores. Since the sample size was limited with respect to number, this may have induced a type II error. In future studies, more specific neuropsychological instruments will be necessary to evaluate cognitive function in schizophrenic patients and healthy subjects.

\section{Acknowledgements}

We would like to acknowledge Dr. Nancy C. Andreasen's research group for their excellent support during the implementation of BRAINS. We thank Prof. Ingrid Agartz (Institute of Psychiatry, University of Oslo, Norway) for some valuable discussions. This work was supported by a grant from the Ministry of Education, Science and Culture of Japan (No. 17790826).

References

-1 Aylward EH, Reiss A, Barta PE, Tien A, Han W, Lee J, Pearson GD: Magnetic resonance imaging measurement of posterior fossa structures in schizophrenia. Am J Psychiatry 1994;151:1448-1452.

2 Joyal CC, Pennanen C, Tiihonen E, Laakso MP, Tiihonen J, Aronen HJ: MRI volumetry of the vermis and the cerebellar hemispheres in men with schizophrenia. Psychiatry Res 2004;131:115-124.

-3 Mathew RJ, Partain CL: Midsagittal sections of the cerebellar vermis and fourth ventricle obtained with magnetic resonance imaging of schizophrenic patients. Am J Psychiatry 1985;142:970-971.

-4 Nasrallah HA, Schwarzkopf SB, Olson SC, Coffman JA: Perinatal brain injury and cerebellar vermal lobules I-X in schizophrenia. Biol Psychiatry 1991;29:567-574. 
5 Nopoulos P, Celley J, Gailis E, Andreasen NC: An MRI study of cerebellar vermis morphology in patients with schizophrenia: evidence in support of the cognitive dysmetria concept. Biol Psychiatry 1999;46:703-711.

6 Rossi A, Stratta P, Mancini F, de Cataldo S, Cassacchia M: Cerebellar vermal size in schizophrenia: a male effect. Biol Psychiatry 1993;33:354-357.

7 Uematsu M, Kaiya H: Cerebellar vermal size predicts drug response in schizophrenic patients: a magnetic resonance imaging (MRI) study. Prog Neuropsychopharmacol Biol Psychiatry 1988;12:837-848.

$\checkmark 8$ Mostofsky SH, Mazzocco MM, Aakalu G, Warsofsky IS, Denckla MB, Reiss AL: Decreased cerebellar posterior vermis size in fragile X syndrome: correlation with neurocognitive performance. Neurology 1998;50: 121-130.

\9 Okugawa G, Sedvall G, Nordström M, Andreasen N, Pierson R, Magnotta V, Agartz I: Selective reduction of the posterior superior vermis in men with chronic schizophrenia. Schizophr Res 2002;55:61-67.

10 American Psychiatric Association: Diagnosis and Statistical Manual of Mental Disorders, ed 4. Washington, American Psychiatric Press, 2000.

-11 Kay SR, Fiszbein A, Opler LA: The Positive and Negative Syndrome Scale (PANSS) for schizophrenia. Schizophr Bull 1987;13:261276.
12 Bell MD, Lysaker H, Milstein RM, BeanGoulet JL: Concurrent validity of the cognitive component of schizophrenia: relationship of PANSS to neuropsychological assessment. Psychiatry Res 1994;54:51-58.

13 Andreasen NC, Cohen G, Harris G, Cizadlo T, Parkkinen J, Rezai K, Swayze VW 2nd: Image processing for the study of brain structure and function: problems and programs. J Neuropsychiatry Clin Neurosci 1992;4:125-133.

14 Andreasen NC, Cizadlo T, Harris G, Swayze VW 2nd, O'Leary DS, Cohen G, Ehrhardt J, Yuh WT: Voxel processing techniques for the antemortem study of neuroanatomy and neuropathology using magnetic resonance imaging. J Neuropsychiatry Clin Neurosci 1993;5:121-130.

15 Harris G, Andreasen NC, Cizadlo T, Bailey JM, Bockholt HJ, Magnotta VA, Arndt S: Improving tissue classification in MRI: a threedimensional multispectral discriminant analysis method with automated training class selection. J Comput Assist Tomogr 1999;23:144-154.

16 Agartz I, Okugawa G, Greiz D, Nordström M, Greitz D, Magnotta VA, Sedvall G: Reliability and reproducibility of brain tissue volumetry from segmented MR scans. Eur Arch Psychiatry Clin Neurosci 2001;251: 255-261.
17 Okugawa G, Takase K, Nobuhara K, Yoshida T, Minami T, Tamagaki C, Magnotta VA, Andreasen NC, Kinoshita T: Inter- and intraoperator reliability of brain tissue measures using magnetic resonance imaging. Eur Arch Psychiatry Clin Neurosci 2003; 253:301-306.

18 Shrout P, Fleiss J: Intraclass correlations: uses in assessing rater reliability. Psychol Bull 1979;86:420-429.

-19 Sullivan EV, Deshmukh A, Desmond JE, Mathalon DH, Rosenbloom MJ, Lim KO, Pfefferbaum A: Contribution of alcohol abuse to cerebellar volume deficits in men with schizophrenia. Arch Gen Psychiatry 2000;57:894-902.

20 Varnäs K, Okugawa G, Hammarberg A, Nesvåg $\mathrm{R}$, Rimol LM, Franck J, Agartz I: Cerebellar volumes in men with schizophrenia and alcohol dependence Psychiatry Clin Neurosci 2007;61:326-329.

21 Okugawa G, Sedvall GC, Agartz I: Smaller cerebellar vermis but not hemisphere volumes in patients with chronic schizophrenia. Am J Psychiatry 2003;160:1614-1617.

22 DeLisi LE, Sakuma M, Taw W, Kushner M, Hoff AL, Grimson R: Schizophrenia as a chronic active brain process: a study of progressive brain structural change subsequent to the onset of schizophrenia. Psychiatry Res 1997;74:129-140.

23 Ichimiya T, Okubo Y, Suhara T, Sudo Y: Reduced volume of the cerebellar vermis in neuroleptic-naïve schizophrenia. Biol Psychiatry 2001;49:20-27. 